\title{
Predation in the Presence of Decoys: An Inhibitory Factor on Pathogen Control by Bacteriophages or Bdellovibrios in Dense and Diverse Ecosystems
}

\author{
Michael H. F. WiLKinSON* \\ Institute for Mathematics and Computing Science, University of Groningen, \\ P.O. Box 800, 9700 AV Groningen, The Netherlands \\ (Received on 11 April 2000, Accepted in revised form on 8 September 2000)

\begin{abstract}
Several attempts have been made at the removal of specific pathogens from the intestinal microflora using either bacteriophages or "predatory" bacteria such as Bdellovibrio spp. To date these attempts have had mixed success. A mechanism explaining these findings based on competitive hindrance by non-prey, or decoy species is put forward. It is shown that this hindrance tends to damp out predator-prey oscillations, and therefore reduces the probability of prey extinction. Possible experiments to verify this theory are discussed. The decoy effect may play a role in any system with high densities of bacteria or other particulate matter, such as activated sludge or biofilms.
\end{abstract}

(C) 2001 Academic Press

\section{Introduction}

The members of the genus Bdellovibrio are obligately predatorial bacteria, which prey on other gram-negative bacteria. Though they are less selective than bacteriophages in their prey selection, each strain preying on a range of gramnegative species (Drutz, 1976; Myamoto \& Kuroda, 1975; Westergaard \& Kramer, 1977), they are by no means generalists. Bdellovibrios are small, aerobic, highly motile curved rods. The free swimming "attack phase" of these bacteria can reach speeds of some $100 \mu \mathrm{m} / \mathrm{s}$, or 70 body lengths per second. They appear to shoot randomly through the medium, and collide with prey species by chance, rather than using chemotaxis (Straley \& Conti, 1977). After colliding with their gram-negative prey, they penetrate the outer membrane and develop in the

\footnotetext{
*E-mail: michael@cs.rug.nl
}

periplasmatic space. The prey cell becomes a rounded "bdelloplast", and finally lyses after the division of the bdellovibrios. This lifestyle bears some similarities to that of bacteriophages, which find their host by diffusion, inject their genetic material, proliferate within the cell and emerge after cell lysis. Bdellovibrios occur in a wide range of habitats, ranging from lowdensity, aquatic, ecosystems (Richardson, 1990; Schoeffield \& Williams, 1990; Rice et al., 1998), to high-density ecosystems such as rhyzosphere soil communities (Elsherif \& Grossmann, 1996) and the gut flora of some vertebrates (Imbragimov, 1980). There are indications that they may associate with surface biofilms in aquatic environments (Kelley et al., 1997). Some sources state that a prey density of at least $10^{4} \mathrm{ml}^{-1}$ is required to support a bdellovibrio population (Rice et al., 1998). However, bdellovibrios may survive starvation periods in stable bdelloplasts (SanchezAmat \& Torrella, 1990). 
In line with other forms of biological pest control, bdellovibrios (Jackson \& Whiting, 1992; Fratamico \& Whiting, 1995) and other predatory bacteria (Casida \& Lukezic, 1992) have been proposed as agents for biological control of pathogenic or food spoilage bacteria. However, the few attempts to control the numbers of gramnegative pathogens using Bdellovibrio spp. in the intestinal microflora have met with no or limited success to date (Imbragimov, 1980; Westergaard \& Kramer, 1977). On the face of it this may seem curious: introducing a predator into an ecosystem in which there is plentiful prey, it fails to colonize. One simple explanation could lie in the aerobic nature of these predatory bacteria (Westergaard \& Kramer, 1977), though survival and growth under anaerobic and microaerobic conditions, respectively, have been reported (Schoeffield et al., 1996). However, obligate aerobes such as Pseudomonas spp. can grow in the intestine, and the isolation of Bdellovibrio bacteriovorus from the intestines and faeces of mammals has been reported (Imbragimov, 1980). In a similar vein, attempts at using lytic phages to eliminate pathogens have met with varying degrees of success, ranging from a total lack of prophylactic effect (Sarkar et al., 1996) to a good measure of protective, and even curative effect during acute diarrhoea (Smith \& Huggins, 1983).

In this article, a mechanism is proposed which may explain some of these observations. The theory is based on interactions between the predator and non-prey species, which coexist with the prey in the ecosystem. Such interactions have been found by Drutz (1976), who observed that $B$. bacteriovorus strains could not predate on Neisseria gonorrhoeae, but that occasional, transient attachment was observed. Furthermore, though the violent collisions seen between predator and prey were not observed between predator and non-prey bacteria, the predators were observed moving around the non-prey cells as if attempting to find a binding site for some time, before "losing interest" in the non-prey cell. The observations of Drutz were made in population densities of $10^{7}-10^{9} \mathrm{ml}^{-1}$. It is shown that at higher densities, such as those found in the gut microflora, this behaviour can have profound consequences on the population dynamics of the predator-prey system.
Of course, non-specialist, protozoan predators thrive in the rumen of livestock (Newbold et al., 1996). Any theory attempting to explain the failure of treatment by specialist predators must also account for the fact that these generalists survive, or even thrive in similar conditions.

Though the gut microflora is highly complex both in its composition and in the fluid dynamics of the system, a chemostat model formalism is used in this paper throughout, for two reasons. Firstly, chemostat models have been used extensively as in vitro models of the gut ecosystem. Though multicompartment and intermittent flow systems perform better than single compartment, constant flow systems, the latter category is capable of reproducing a number of important properties of especially the luminal flora of the large intestine (Nisbet et al., 1993; Nuotio \& Mead, 1993; Marteau et al., 1997). Since this is the compartment with the highest densities, this is the most important compartment to model well in view of the current model. A similar situation is seen in the very few computer simulation models of the gut ecosystem, where chemostatlike models (Freter et al., 1983; Coleman et al., 1996) are used in all cases but the MIMICS simulator developed in our department (Wilkinson, 1997), which uses a reaction/diffusion system with bulk motion included.

The second, more important reason for using the chemostat formalism is that analytical results can be obtained easily, and stability analysis is straightforward. The partial differential equations involved in the reaction/diffusion/bulkmotion system used in the MIMICS simulator should give a more accurate simulation in the gut microflora case, but analytical solutions cannot be obtained.

\section{The Decoy Mechanism}

PREDATION IN THE PRESENCE OF DECOYS

In the following discussion, we will use the double-Monod model (Canale, 1969) with and without extensions for maintenance energy costs (Nisbet et al., 1983) to study the effect of decoys on the dynamics of predator-prey interactions. Though the dynamic energy budget model (Kooi \& Kooijman, 1994a, b) models certain features of 
microbial predator-prey system more accurately, the models of uptake of prey by predators (by Michaelis-Menten terms) are identical for both models. As will be shown, the effect of decoys lies in altering the Michaelis-Menten constant of this uptake process, so the impact of this effect should be similar in both models. Besides, the model of Kooi and Kooijman (1994a), like that of Jost et al. (1973), is designed to take the size distribution of the predator into account. They pertain to predators larger than their prey, which divide into smaller (juvenile) cells after reaching a certain size. Bdellovibrios and bacteriophages are smaller than their hosts, and divide inside the host, emerging as full-grown predators. Therefore, the population size structure is not expected to be important. In the case of bdellovibrios, maintenance energy must be taken into account, but not in the case of bacteriophages. Therefore, I use the simpler double-Monod model, with extensions with maintenance energy as in Nisbet et al. (1983). The model of Marchand \& Gabignon (1981) for the B. bacteriovorus-Escherichia coli system differs from the models discussed above, in which they model infected and noninfected prey cells explicitly. This will also be done in the discussion of the decoy effect. However, Marchand and Gabignon do not introduce a third species into the interaction.

Thus, the growth of microbial predators $Y$ on prey species $X_{1}$ is modelled as

$$
\frac{\mathrm{d} Y}{\mathrm{~d} t}=\frac{\mu_{y} X_{1}}{K_{X}+X_{1}} Y-\left(D+d_{y}\right) Y
$$

in which $\mu_{y}$ is the maximum specific growth rate, $K_{X}$ the saturation constant, $D$ the dilution rate of the chemostat, and $d_{y}$ the starvation rate, or maintenance energy term (Nisbet et al., 1983). The differential equation for species $X_{1}$ is

$$
\frac{\mathrm{d} X_{1}}{\mathrm{~d} t}=\frac{\mu_{1} X_{0}}{K_{1}+X_{0}} X_{1}-\frac{V_{y} X_{1}}{K_{X}+X_{1}} Y-D X_{1},
$$

in which $X_{0}$ is the concentration of the limiting substrate, $\mu_{1}$ the maximum specific growth rate, $K_{1}$ the saturation constant, and $V_{y}$ the maximum specific uptake rate of prey by predator. The differential equation for the limiting substrate becomes

$$
\frac{\mathrm{d} X_{0}}{\mathrm{~d} t}=D\left(S-X_{0}\right)-V_{1} \frac{X_{0}}{K_{1}+X_{0}} X_{1}
$$

in which $S$ is the input substrate concentration, and $V_{1}$ the maximum specific uptake rate of $X_{0}$ by $X_{1}$.

We will now take the case of a three-species ecosystem: one prey species $X_{1}$, one non-prey species $X_{2}$, and a predator $Y$. Species $X_{2}$ is not modelled explicitly using differential equations of its own, since we assume it uses a different limiting substrate than species $X_{1}$.

This is done to study the effect of the simple presence of a "decoy" species, independent of any other competition effect. Though the word "decoy" might be thought of as implying attraction of the predator or parasite species, this is not intended. The presence of the decoy species simply leads to collisions with the predator, which is assumed to move through the medium randomly.

Disregarding starvation, the predator can be in three states: free, bound to $X_{1}$, and bound to $X_{2}$. These complexes are denoted as $\left[X_{1} Y\right]$ and $\left[X_{2} Y\right]$. Assume the rate of collisions is $r$ per unit of prey or non-prey species per unit of predator. Since chemotaxis towards prey has not been observed in Bdellovibrio spp. (Straley \& Conti, 1977) and bacteriophages are not motile, this rate is assumed to be identical for prey and non-prey species. Furthermore, the prey/predator complex dissociates at a rate of $k_{1}$, and non-prey/predator complex dissociates at a rate of $k_{2}$. However, only the dissociation of the first complex yields new predators, with a yield of $y_{x}+1$. Since one phage or predator is lost in the forming of this complex, the net yield per unit of prey biomass is $y_{x}$.

The central assumption of this theory is that after colliding with a non-prey cell, a predator will briefly attach, before detaching, as observed in $N$. gonorrhoeae-B. bacteriovorus systems (Drutz, 1976). The rate constant $k_{2}$ will therefore be finite. We also assume that all reaction rates are an order of magnitude higher than the dilution rate of the entire system, for the sake of quasi-steady-state analysis. Since the dilution rate of the large intestine is in the order of one per 
day, this assumption should hold reasonably well there. This leads to the following set of differential equations:

$$
\begin{gathered}
\frac{\mathrm{d} Y_{\text {free }}}{\mathrm{d} t}=\left(y_{x}+1\right) k_{1}\left[X_{1} Y\right]+k_{2}\left[X_{2} Y\right] \\
-r\left(X_{1}+X_{2}\right) Y_{\text {free }}, \\
\frac{\mathrm{d}\left[X_{1} Y\right]}{\mathrm{d} t}=-k_{1}\left[X_{1} Y\right]+r X_{1} Y_{\text {free }}, \\
\frac{\mathrm{d}\left[X_{2} Y\right]}{\mathrm{d} t}=-k_{2}\left[X_{2} Y\right]+r X_{2} Y_{\text {free }} .
\end{gathered}
$$

The term at steady state is

$\left[X_{1} Y\right]=\frac{r}{k_{1}} X_{1} Y_{\text {free }} \quad$ and $\quad\left[X_{2} Y\right]=\frac{r}{k_{2}} X_{2} Y_{\text {free }}$

Remembering that the total predator content $Y$ is

$$
\begin{aligned}
Y & =\left[X_{1} Y\right]+\left[X_{2} Y\right]+Y_{\text {free }} \\
& =\left(1+\frac{r}{k_{1}} X_{1}+\frac{r}{k_{2}} X_{2}\right) Y_{\text {free }}
\end{aligned}
$$

and summing eqns $(4 a-c)$ we find a growth rate of

$$
\begin{aligned}
\frac{\mathrm{d} Y}{\mathrm{~d} t} & =\frac{y_{x} k_{1} X_{1} Y}{k_{1} / r+X_{1}+k_{1} X_{2} / k_{2}} \\
& =\frac{\mu_{y} X_{1} Y}{K_{X}+X_{1}+K_{i n h} X_{2}}
\end{aligned}
$$

in which we recognize the standard form of competitive inhibition. Therefore, in an extremely densely populated and diverse ecosystem, such as the intestinal microflora, specialist predators would be in a serious disadvantage compared to generalists.

The reasoning (and differential equations) used above can be used without adaptation for lytic bacteriophages.

\section{THE CONSEQUENCES FOR THE ECOSYSTEM}

In the following discussion we use the differential equations given by eqns (1)-(3). The decoy species is not considered to be a competitor of the prey, so its actions can be absorbed into the saturation constant

$$
K_{X}^{*}=K_{X}+K_{i n h} X_{2}
$$

This set of differential equations was studied in detail by Canale (1969). Four different "modes" of operation can be identified (Kooi \& Kooijman, 1994a): (0) total washout of both species; (I) stable prey population with washout of predator; (II) stable coexistence of predator and prey, and (III) unstable coexistence (limit-cycle behaviour). If both species coexist, the (stable or unstable) focus is given by

$$
\begin{aligned}
& X_{0}= \frac{1}{2}\left(S-K_{1}-\frac{V_{1} K_{X}^{*}}{\mu_{y}-D-d_{y}}\right. \\
&\left. \pm \sqrt{\left(S-K_{1}-\frac{V_{1} K_{X}^{*}}{\mu_{y}-D-d_{y}}\right)^{2}+4 K_{1} S}\right) \\
& X_{1}=\frac{\left(D+d_{y}\right) K_{X}^{*}}{\mu_{y}-D-d_{y}}, \\
& Y=\frac{D}{D+d_{y}} \frac{\mu_{y}}{V_{y}}\left(\frac{\mu_{1}}{V_{1}}\left(S-X_{0}\right)-X_{1}\right) .
\end{aligned}
$$

Thus, it can be seen that the equilibrium concentration of prey is directly proportional to the saturation constant $K_{X}^{*}$, and should therefore vary linearly with the concentration of decoys (of course, provided that the solution of $Y$ is positive). The boundaries between modes 0 and I and between I and II as a function of the chemostat's control parameters (dilution rate $D$ and input concentration of the limiting substrate $S$ ) can be obtained analytically. The first boundary is given by

$$
S=\frac{D K_{1}}{\mu_{1}-D}
$$

The boundary between (I) and (II) is

$$
S=\frac{D K_{1}}{\mu_{1}-D}+\frac{V_{1} K_{X}^{*}}{\mu_{1}} \frac{D+d_{y}}{\mu_{y}-D-d_{y}} .
$$



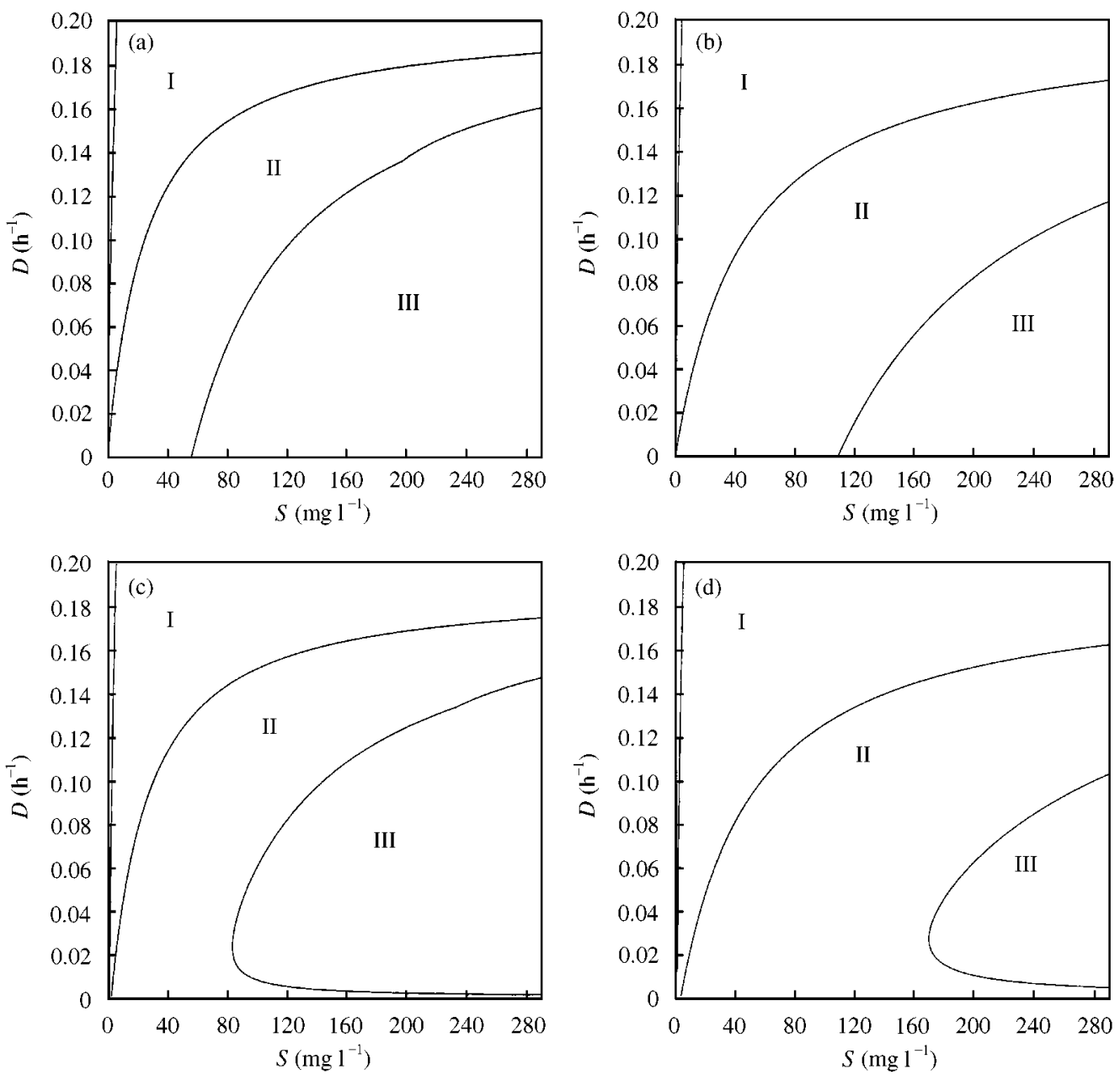

FIG. 1. Operating diagrams for chemostats containing microbial predator-prey systems using the double Monod model with different number of decoys: (a): double Monod model without maintenance energy, using $K_{X}=K_{X}^{*}=9$; (b) same as (a) but with $K_{X}^{*}=18$; (c) and (d): same as (a) and (b), but with maintenance energy term $d_{y}=0.01$. In zone I, only prey survive, in zone II both predator and prey coexist in a stable equilibrium, and in zone III the system becomes unstable. Increasing $K_{X}^{*}$ reduces zone III and increases zone I.

As can be seen, increasing $K_{X}^{*}$ means that, for a given $D$, the predator can only be present in the ecosystem at all at a higher input substrate concentration than in the absence of decoys. Figure 1 shows the zone boundaries as a function of the control parameters, using the same values for the metabolic parameters of predator and prey as were used by Nisbet et al. (1983) and Kooi \& Kooijman (1994a) (i.e. $K_{1}=8 \mathrm{mg}^{-1}$, $K_{X}=9 \mathrm{mgl}^{-1}, \quad \mu_{1}=0.5 \mathrm{~h}^{-1}, \quad \mu_{y}=0.2 \mathrm{~h}^{-1}$, $V_{1}=1.25 \mathrm{~h}^{-1}, \quad$ and $\quad V_{y}=0.3333 \mathrm{~h}^{-1}$ ). The zone boundary between zones II and III was obtained by local stability analysis of the steady-state solution. Figures 1(a) and (b) show the results for the double Monod model without metabolic costs, whereas 1(c) and (d) show the results for $d_{y}=0.01 \mathrm{~h}^{-1}$. Figures 1(b) and (d) show the stabilizing effect of decoys, with $K_{X}^{*}=2 K_{X}$.

Figure 2 shows the transient dynamical behaviour of these systems when a small number of predators are introduced in a system in which the prey is present at its equilibrium value in the absence of predators, with $D=0.1 \mathrm{~h}^{-1}$ and $S=200 \mathrm{mg}^{-1}$. The same parameters were used as in Fig. 1. The damping effect of decoys on the predator-prey oscillations is evident. In the absence of decoys, the prey is almost driven to extinction. In practice, total extinction may occur. By contrast, if $K_{X}^{*}=2 K_{X}$, the prey is only 

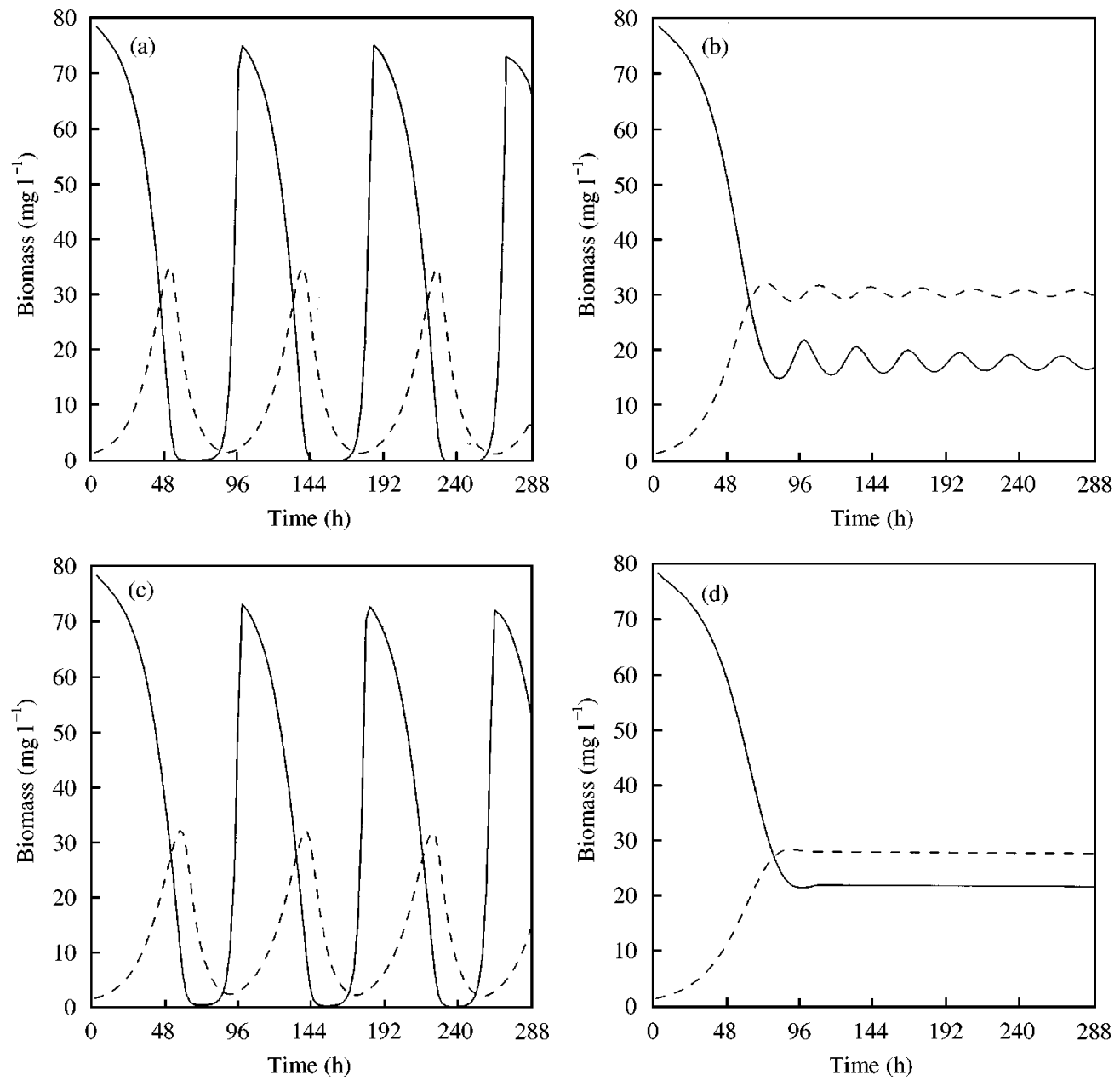

FIG. 2. Transient behaviour of chemostats containing microbial predator-prey systems using the double Monod model with different number of decoys: (a): double Monod model without maintenance energy, using $K_{X}=K_{X}^{*}=9$; (b) same as (a) but with $K_{X}^{*}=18$; (c) and (d): same as (a) and (b), but with maintenance energy term $d_{y}=0.01$. In the absence of decoys, the prey is driven to near extinction, whereas the presence of decoys leads to a reduction of only a factor of 3.6 (or $0.56 \mathrm{on} \mathrm{a}^{10} \log$ scale): $(-)$ prey; $(----)$ predator.

reduced by a factor of 3.6 or $0.56{ }^{10} \log$ steps, so prey extinction does not occur.

\section{ESTIMATING THE MAGNITUDE OF THE EFFECT}

One shortcoming of the theory presented here so far is that no estimate of the magnitude of the inhibitory effect can be given a priori. In the case of actively swimming predators such as Bdellovibrios, a lower limit can be given. Assume that collisions with decoys are completely non-elastic, and that the decoys are far more massive than the predator. Furthermore, assume that the predator immediately starts a tumble (without any time needed for signal transduction). A typical tumble lasts in the order of $0.1 \mathrm{~s}$ (Singleton, 1995), yielding a value of $10 \mathrm{~s}^{-1}$ for dissociation constant $k_{2}$. Furthermore, we can estimate the collision rate constant $r$ as

$$
r=A_{\text {col }} v_{\text {pred }}
$$

in which $v_{\text {pred }}$ is the mean swimming velocity of the predator, and $A_{c o l}$ is the collisional crosssection. Setting $A_{c o l}$ to $1 \mu \mathrm{m}^{2}$ and $v_{\text {pred }}$ to $100 \mu \mathrm{m} \mathrm{s}^{-1}$, we find $r=100 \mu \mathrm{m}^{3} \mathrm{~s}^{-1}$, or $1 \times 10^{-10}$ $\mathrm{cm}^{3} \mathrm{~s}^{-1}$. Writing the effective Michaelis-Menten 
constant as

$$
K_{X}^{*}=k_{1}\left(1 / r+\left[X_{2}\right] / k_{2}\right)
$$

we find that at a decoy concentration of $10^{11} \mathrm{~cm}^{-3}, K_{X}^{*}$ has doubled compared to the case without decoys. Since the number of bacteria in the large intestine can be in the order of $3 \times 10^{11} \mathrm{~cm}^{-3}$, the effect of decoys may be appreciable. Remember that this is a lower limit of the magnitude of the effect. Drutz (1976) noted that bdellovibrios swim around $N$. gonorrhoeae cells as if trying to find a suitable point of entry, and occasionally transient attachment was observed. Unfortunately, for our purposes, he did not measure the time bdellovibrios took to "investigate" the non-prey cells, but we may assume it would be in the order of $1 \mathrm{~s}$ at least for it to be described as Drutz does. This means that the effect can be 10 times larger than the above estimate, and doubling of $K_{X}^{*}$ should take place at a concentration of $10^{10} \mathrm{~cm}^{-3}$, and the $K_{X}^{*}$ in the large intestine could be 30 times greater than that measured in the absence of decoys. Using an intermediate value of $K_{X}^{*}=10 K_{X}$, and the same parameters as in Fig. 2, the prey (pathogen) would only be reduced by $1.1 \%$.

For bacteriophages the situation is different. In this case, the collision rate is limited by diffusion, rather than active propulsion. It will most certainly be an order of magnitude lower than in the case of bdellovibrio. Estimating the dissociation constant $k_{2}$ is also problematic. However, if we assume the collisions are inelastic, and some nonspecific binding takes place, it might become sufficiently large to have an effect similar to the case of bdellovibrios. I am not aware of any experiments which provide direct evidence of transient binding of phages to non-prey bacteria, but it is unlikely that no such binding occurs.

\section{Discussion}

A PROPOSAL FOR TESTING THE HYPOTHESIS

A continuous flow co-culture of E. coli serving as prey, Bdellovibrio bacteriovorus as predator, and some aerotolerant Lactobacillus sp. as decoy could be an ideal model system to measure the proposed decoy effect. Lactobacilli are grampositive, and therefore immune to the attack from B. bacteriovorus. Besides, E. coli and the lactobacilli can be grown without direct competition if glucose and lactose are used as limiting substrates for the two species, respectively. Alternatively, heat-killed bacteria of a suitable gram-positive species could be added at various concentration to the inflowing medium. Given the observations of Drutz (1976), N. gonorrhoeae would seem an ideal candidate. The steady-state prey population should vary linearly with the decoy population according to eqn (7b).

\section{CONCLUSIONS}

The theory presented in this paper may go some way to explain certain failures of pathogen elimination by specialist predators, and possibly bacteriophages in the intestine. The theory does allow colonization of the intestinal flora, and some reduction in the prey should occur in such cases. More importantly from a therapeutic point of view, the ecosystem becomes more stable by the presence of decoys, so that the oscillatory behaviour needed to drive the prey to (near) extinction is reduced. Only when the prey (or host) is abundant compared to other species can the predators such as B. bacteriovorus or lytic phages reach the same levels at steady state as in the absence of decoys. The protective and curative effect of phage treatment obtained by Smith and Huggins (1983), can be explained in this way, since their experimental animals were infected between 6 and $16 \mathrm{hr}$ after birth, by which time no complex intestinal microflora is expected to be present yet, and pathogens as E. coli may rapidly dominate (Schaedler, 1972). Besides, during acute bacterial diarrhoea, large numbers of pathogens may be present, and dilution of the ecosystem occurs (Miskovitz \& Rochwarger, 1993).

A large number of alternative mechanisms for the failure of pathogen control by bdellovibrios or phages can be put forward (Westergaard \& Kramer, 1997). They include the stomach acidity, anaerobic nature of the intestine, the action of enzymes, and adhesion of immunoglobulins, especially IgA. All these factors, and possibly many more may indeed play a role in limiting the success of biological pathogen control in the gut. 
In the case of Bdellovibrio spp. immunoglobulins in particular may cause problem, since these species incorporate complete cell wall structures and membrane protein fragments of the prey in their own outer envelope (Diedrich, 1988; Stein et al., 1992). If the host has mounted an immune response to the pathogen (prey) it is likely to affect the predator as well in such cases. It is harder to see why stomach acidity should have an effect, since Westergaard \& Kramer (1977) successfully administer bdellovibrios orally. Also, the anaerobic environment of the gut should not affect bacteriophages.

The effect described here is the opposite of the "alternative prey" effect observed by Mallory et al. (1983). This effect occurs when the predator is not too specific. Mallory et al. studied the elimination of salmonellas by predators in sewage. They found that addition of an alternative, harmless prey species to the ecosystem caused increased predation of the salmonellas. This is explained by assuming that the alternative prey increased the mean number of more-or-less generalist predators. Quite contrary to the introduction of decoys, addition of alternative prey should also lead to an increased likelihood of Lottka-Volterra oscillations, as is often observed in more hypertrophic systems (DeAngelis, 1992). However, adding generalist predators to the intestinal microflora for therapeutic reasons may have an effect similar to broad spectrum antibiotics, and cause overgrowth by resistant pathogens.

The situation may be different for non-obligate predators, similar to Pseudomonas strain 679-2 (Casida, 1992). If suitable, non-obligate, specialist predators could be supported in sufficiently large numbers by nutrients in the intestine, they may be able to reduce the number of bacteria of certain species in the intestine effectively.

In the previous discussion, the decoy effect is attributed only to other bacteria. However, other particulate matter such as fibre could also act as decoy. Furthermore, this effect should not be limited to the gut microbial ecosystem. The decoy effect may play a role in any system with high densities of bacteria, such as activated sludge or biofilms, or indeed to other predator-prey systems altogether. In any system, where a predator may be hindered in any way by the presence of another, inedible species, the decoy effect will come into effect.

This study was made possible by generous funding provided by the Institute for Microbiology, HerbornDill, Germany, and the International Study Group for New Antimicrobial Strategies (ISGNAS).

\section{REFERENCES}

Canale, R. P. (1969). Predator-prey relationships in a model for the activated sludge process. Biotech. Bioeng. XI, 887-907.

Casida, L. E. Jr. \& Lukezic, F. L. (1992). Control of leaf spot diseases of alfalfa and tomato with application of the bacterial predator Pseudomonas strain 679-2. Plant Dis. 76, 1217-1220.

Coleman, M. E. Dreesen, D. W. \& Wiegert, R. G. (1996). A simulation of microbial competition in the human colonic ecosystem. Appl. Environ. Microbiol. 62, 3632-3639.

DeANGELIS, D. L. (1992). Dynamics of Nutrient Cycling and Food Webs, pp. 81-87. London: Chapman \& Hall.

DIEDRICH, D. L. (1988). Bdellovibrios: recycling, remodelling and relocalizing components from their prey. Microbiol. Sci. 5, 100-103.

DRUTZ, D. J. (1976). Response of Neisseria gonorrhoea to Bdellovibrio species. Infect. Immun. 13, 247-251.

Elsherif, M. \& Grossmann, F. (1996). Role of biotic factors in the control of soil-borne fungi by fluorescent pseudomonads. Microbiol. Res. 151, 351-357.

Fratamico, P. M. \& Whiting, R. C. (1995). Ability of Bdellovibrio bacteriovorus 109J to lyse gram-negative food-borne pathogenic and spoilage bacteria. J. Food Prot. 58, 160-164.

Freter, R., Brickner, H., Fekete, J., Vickerman, M. M. \& CAREY, K. V. (1983). Survival and implantation of Escherichia coli in the intestinal tract. Infect. Immun. 39, 686-703.

Imbragimov, F. K H. (1980). Obsemenie bakteriiami Bdellovibrio bacteriovorus organizma zhivotnykh i ikh vzaimodeistvie s vozbuditeliami ostrykh kishechnykh infektsii. Zh. Mikrobiol. Epidemiol. Immunobiol. 5, 97-99.

JACKSON, L. \& WhiTING, R. C. (1992). Reduction of an Escherichia coli K12 population by Bdellovibrio bacteriovorus under various in vitro conditions of parasite: host ratio, temperature, or pH. J. Food Prot. 55, 859-861.

Jost, J. L., Drake, J. F., Tsuchiya, H. M. \& FrederickSON, A. G. (1973). Microbial food chains and food webs. J. theor. Biol. 41, 461-484.

Kelley, J. I., Turng, B. F., Williams, H. N. \& Baer, M. L. (1997). Effects of temperature, salinity, and substrate on the colonization of surfaces in situ by aquatic Bdellovibrios. Appl. Environ. Microbiol. 63, 84-80.

Kool, B. W. \& Kooljman, S. A. L. M. (1994a). Existence and stability of microbial prey-predator systems. J. theor. Biol. 170, 75-85.

Kool, B. W. \& Kooljman, S. A. L. M. (1994b). The transient behaviour of food chains in chemostats. J. theor. Biol. 170, 87-94.

Mallory, L. M., YuK, C. S., LiAnG, L. N. \& AleXANDer, M. (1983). Alternative prey: a mechanism for elimination of bacterial species by protozoa. Appl. Environ. Microbiol. 46, 1073-1079. 
Marchand, A. \& Gabignon, O. (1981). Modele theoretique de la cinetique d'interaction du couple proie-predateur Bdellovibrio bacteriovorus-Escherichia coli. Ann. Microbiol. (Paris) 132 B(3), 321-326.

Marteau, P., Minekus, M., Havenaar, R. \& Huis in'T VELD, J. H. (1997). Survival of lactic acid bacteria in a dynamical model of the stomach and the small intestine: validation and the effect of bile. J. Dairy Sci. 80, 1031-1037.

Miskovitz, P. F. \& Rochwarger, A. M. (1993). The Evaluation and Treatment of the Patient with Diarrhoea, pp. 45-96. Stoneham, MA: Butterworth-Heinemann.

MyAmoto, S. \& KurodA, K. (1975). Lethal effect of fresh sea water on Vibrio parahaemolyticus and isolation of Bdellovibrio parasitic against the organism. Jpn. J. Microbiol. 19, 309-317.

Newbold, C. J., Ushida, K., Morvan, B., Fonty, G. \& JoUAnY, J. P. (1996). The role of ciliate protozoa in the lysis of methanogenic archaea in rumen fluid. Lett. Appl. Microbiol. 23, 421-425.

Nisbet, R. M., Cunningham, A. \& Gurney, W. S. C. (1983). Endogenous metabolism and the stability of microbial prey-predator systems. Biotechnol. Bioeng. XXV, 301-306.

Nisbet, D. J., Corrier, D. E. \& DeLoach, J. R. (1993). Effect of mixed cecal microflora maintained in continuous culture and of dietary lactose on Salmonella typhimurium colonization in broiler chicks. Avian Dis. 37, 528-535.

Nuotio, L. \& Mead, G. C. (1993). An in vitro model for studies on bacterial interactions in the avian caecum. Lett. Appl. Microbiol. 17, 65-67.

Rice, T. D., Williams, H. N. \& Turng, B. F. (1998). Susceptibility of bacteria in estuarine environments to autochtenous bdellovibrios. Microb. Ecol. 35, 256-264.

RICHARDSON, I. R. (1990). The incidence of Bdellovibrio spp. in man-made water systems: coexistence with legionellas. J. Appl. Bacteriol. 69, 134-140.

SAnchez-Amat, A. \& Torella, F. (1990). Formation of stable bdelloplasts as a starvation-survival strategy of marine bdellovibrios. Appl. Environ. Microbiol. 56, 2717-2725.

Sarkar, B. L., Chakrabarti, A. K., Koley, H., ChaKRABARTI, M. K. \& DE, S. P. (1996). Biological activity and interaction of Vibrio cholerae bacteriophages in rabbit ileal loop. Indian J. Med. Res. 104, 139-141.

SCHAEDLER, R. W. (1972). Symposium on 'Gut microflora and nutrition in the non-ruminant', the relationship between the host and its intestinal microflora. Proc. Nutr. Soc. 32, 41-47.

Schoeffield, A. J. \& Williams, H. A. (1990). Efficiencies of recovery of bdellovibrios from brackish-water environments by using various bacterial species as a prey. Appl. Environ. Microbiol. 56, 230-236.

Schoeffield, A. J., Huggins, H. N., Turng, B. \& FACKLER, W. A. Jr. (1996). A comparison of the survival of intraperiplasmic and attack phase Bdellovibrios with reduced oxygen. Microbiol. Ecol. 32, 35-46.

Sharp, R., Hazlewood, G. P., Gilbert, H. J. \& O'DonNELL, A. G. (1994). Unmodified and recombinant strains of Lactobacillus plantarum are rapidly lost from the rumen by protozoal predation. J. Appl. Bacteriol. 76, 110-117.

Singleton, P. (1995). Bacteria in Biology, Biotechnoloy, and Medicine, p. 23. Chichester, UK: Wiley.

Smith, H. W. \& HugGins, M. B. (1983). Effectiveness of phages in treating experimental Escherichia coli diarrhoea in calves piglets and lambs. J. Gen. Microbiol. 129, 2659-2675.

Stein, M. A., McAllister, S. A., Torian, B. E. \& DiedRICH, D. L. (1992). Acquisition of apparently intact and unmodified lipopolysaccharides from Escherichia coli by Bdellovibrio bacteriovorus. J. Bacteriol. 174, 2858-2864.

Straley, S. C. \& Conti, S. F. (1977). Chemotaxis by Bdellovibrio bacteriovorus toward prey. J. Bacteriol. 132, 628-640.

Wallace, R. J. \& MCPherson, C. A. (1987). Factors affecting the rate of breakdown of bacterial protein in rumen fluid. Br. J. Nutr. 58, 313-323.

WestergaArD, J. M. \& KrAmer, T. T. (1977). Bdellovibrio and the intestinal flora of vertebrates. Appl. Environ. Microbiol. 34, 506-511.

WiLKinson, M. H. F. (1997). Nonlinear dynamics, chaostheory, and the "sciences of complexity": their relevance to the study of the interaction between host and microflora. In: Old Herborn University Monograph, Vol. 10: New Antimicrobial Strategies (Heidt, P. J., Rusch, V. \& Van der Waaij, D., eds), pp. 111-130. Herborn-Dill, Germany: Herborn Litterae.

\section{Appendix \\ List of Symbols}

Species

$X_{0}$

$X_{1}$

$X_{2}$

Y

$Y_{\text {free }}$

$\left[X_{1} Y\right],\left[X_{2} Y\right]$

Parameters

$A_{\text {col }}$

$D$

$d_{y}$

$K_{1}$

$K_{X}$

$K_{X}^{*}$

$K_{i n h}$ concentration of limiting substrate for $X_{1}$

prey species concentration

non-prey, or decoy species concentration

total predator species concentration

free predator species concentration

concentrations of complexes of predator bound to $X_{1}$ and $X_{2}$

collisional cross-section

dilution rate

starvation rate, or maintenance energy term for species $Y$ Michaelis-Menten constant for growth of $X_{1}$ on $X_{0}$ Michaelis-Menten constant for growth of $Y$ on $X_{1}$ in the absence of $X_{2}$

Michaelis-Menten constant for growth of $Y$ on $X_{1}$ in the presence of $X_{2}$ inhibition constant due to decoy effect 


\begin{tabular}{|c|c|c|c|}
\hline$k_{1}, k_{2}$ & $\begin{array}{l}\text { dissociation rate constants } \\
\text { of complexes }\left[X_{1} Y\right] \text { and } \\
{\left[X_{2} Y\right]}\end{array}$ & $\begin{array}{l}S \\
V_{1}\end{array}$ & $\begin{array}{l}\text { input concentration of } X_{0} \\
\text { maximum specific uptake rate } \\
\text { of } X_{0} \text { by } X_{1}\end{array}$ \\
\hline$\mu_{1}$ & $\begin{array}{l}\text { maximum specific growth rate } \\
\text { of } X_{1} \text { on } X_{0}\end{array}$ & $V_{y}$ & $\begin{array}{l}\text { maximum specific uptake rate } \\
\text { of } X_{1} \text { by } Y\end{array}$ \\
\hline$\mu_{y}$ & $\begin{array}{l}\text { maximum specific growth rate } \\
\text { of } Y \text { on } X_{1} \\
\text { collision rate constant }\end{array}$ & $\begin{array}{l}v_{\text {pred }} \\
y_{x}\end{array}$ & $\begin{array}{l}\text { mean swimming velocity of } Y \\
\text { net yield of } Y \text { biomass per unit } \\
\text { of } X_{1}\end{array}$ \\
\hline
\end{tabular}

